\title{
El papel de la universidad frente al abandono de estudios. Formación pre-universitaria basada en talleres de innovación y creatividad
}

\author{
The role of the university against the abandonment of studies. \\ Pre-university training based on innovation and creativity workshops
}

Nuria Nebot Gómez de Salazar

RECIBIDO 15/3/2019 ACEPTADO 20/3/2020 PUBLICADO 1/6/2020

Departamento Arte y Arquitectura, Universidad de Málaga, España

nurianebot@uma.es

Antonio Álvarez Gil

Departamento Arte y Arquitectura, Universidad de Málaga, España

aalvarez@uma.es

RESUMEN

Uno de los retos a los que se enfrentan muchos jóvenes al terminar sus estudios de formación secundaria o ciclos formativos es la elección de una carrera universitaria. La elevada tasa de abandono de los estudios universitarios en España, especialmente en los primeros cursos académicos, es el reflejo de elecciones inadecuadas y desajustes entre las expectativas creadas y la realidad con que se encuentra el alumnado. Según datos del Ministerio de Educación, Cultura y Deporte, el abandono de los estudios afecta a casi un 20\% del total de los estudiantes (curso académico 2013-2014) lo que revela la necesidad de atender esta situación, buscando soluciones y nuevas medidas preventivas.

Son muchas las investigaciones que han analizado las causas de dicho abandono universitario tratando de establecer unas medidas de prevención que sean eficaces. Muchos de estos estudios coinciden en la importancia del papel de la propia universidad en la preparación de la inserción de estudiantes de bachillerato o ciclos formativos.

Esta investigación tiene como objetivo prioritario profundizar en la colaboración entre centros de educación secundaria y universitarios, incidiendo en nuevos modelos colaborativos de innovación y creatividad en la etapa previa a la entrada en la universidad. La aportación más importante se traduce en mostrar una experiencia de talleres prácticos e interactivos entre alumnado de altas capacidades de centros escolares y alumnado y profesorado de la titulación de Arquitectura.

Los objetivos de los talleres son introducir al estudiante en el campo de conocimiento de la Arquitectura, explicar la importancia de los estudios en la sociedad actual y despertar vocaciones entre otros. Para ello, se ha llevado a cabo una metodología basada en talleres interactivos que fomentan el trabajo en equipo y la creatividad del alumnado. Los resultados de la experiencia han sido satisfactorios, tanto la valoración de la encuesta realizada a los participantes como la valoración de los propios mentores del programa. Las conclusiones subrayan la importancia del cambio de modelo en la formación pre-universitaria: desde las actuales sesiones informativas, donde el alumnado es sujeto pasivo, hacia una formación en la que el alumnado se convierte en agente activo en el proceso de crear conocimiento.

PALABRAS CLAVE abandono de estudios, formación pre-universitaria, colaboración entre universidad y centros de educación secundaria, talleres interactivos, creatividad, innovación, arquitectura. 


\section{ABSTRACT}

The choice of the university studies becomes one of the problems that many young people face when they finish their secondary education or training cycles. The high dropout rate of university studies in Spain, especially in the first academic years, reflects possible inadequate choices and imbalances between the expectations created and the reality the students find. According to data from the Spanish Ministry of Education, Culture and Sports, the abandonment of studies affects almost 20\% of the total of students (academic year 2013-2014) which reveals the need to address this situation, by seeking solutions and new preventive measures.

There are many researches that have analyzed the causes of this university dropout trying to establish effective prevention measures. Many of these studies agree on the importance of the role of the university itself in the preparation of the insertion of high school students or training cycles.

The main objective of this research is to investigate the possibilities of collaboration between secondary education centers and university centers, focusing on new collaborative models of innovation and creativity in the stage prior to entering university. The most important contribution is translated into showing a real experience of practical and interactive workshops between high school students with high capacities and students and professors of the Architecture degree.

The objectives of the workshops are the following ones: firstly, to introduce the student to the field of knowledge of Architecture. Secondly, to explain the importance of studies in today's society and, thirdly, to awaken vocations among others. In order to achieve these goals, a methodology based on interactive workshops that promote teamwork and student creativity has been carried out. The results of this experience have been satisfactory, both the evaluation of the survey made to the participants and the evaluation of the mentors of the program.

KEYWORDS abandonment of studies, pre-university training, collaboration between university and secondary schools, interactive workshops, creativity, innovation, architecture.

\section{INTRODUCCIÓN}

Uno de los retos a los que se enfrentan muchos jóvenes al terminar sus estudios de formación secundaria o ciclos formativos es la elección de una carrera universitaria. La elevada tasa de abandono de los estudios universitarios en España, especialmente en los primeros cursos académicos, es el reflejo de elecciones inadecuadas y desajustes entre las expectativas creadas y la realidad con que se encuentra el alumnado. Según datos del Ministerio de Educación, Cultura y Deporte, el abandono de los estudios afecta a casi un 20\% del total de los estudiantes (curso académico 2013-2014) lo que revela la necesidad de atender esta situación, buscando soluciones y nuevas medidas preventivas (Datos básicos del sistema universitario español. Curso 2013-2014. Catálogo de publicaciones del Ministerio: mecd.gob.es, 2013).

Son muchas las investigaciones que han analizado las causas de dicho abandono universitario tratando de establecer unas medidas de prevención que sean eficaces. Conocer los aspectos que inducen al abandono permite a los docentes y a la propia universidad trabajar con aquéllos a su alcance. Resulta de especial interés la investigación realizada por Corominas Rovira en la que, a través de cuestionarios, recoge información directa de estudiantes que han abandonado los estudios, señalando tres causas principales: carencia en la calidad de enseñanza de la institución, déficits en el potencial de aprendizaje del alumno y dudas sobre la elección pertinente de los estudios (Corominas Rovira, 2001).

Araque et al. (2009) señalan factores como la edad de ingreso, el nivel educativo de los padres, el éxito académico en etapas educativas preuniversitarias y el bajo rendimiento académico como variables más 
influyentes. A su vez, Willcoxson (2010) destaca, como factores determinantes, aspectos personales tales como la falta de compromiso con la institución o la falta de integración social, así como otros de carácter académico relacionados con las habilidades, el apoyo del personal y las expectativas de aprendizaje.

Bernardo et al. (2015) evidencian la relación entre abandono de titulación universitaria con tres aspectos: en primer lugar, el rendimiento académico previo del alumnado (en términos de nota de acceso a la universidad), lo que les lleva a proponer medidas de recomendación tales como el seguimiento del alumnado con calificaciones más bajas en etapas previas al ingreso. En segundo lugar, establecen una relación directa entre el fenómeno del abandono y el ingreso retrasado en la titulación, una vez iniciado el periodo lectivo. $\mathrm{Y}$, en tercer lugar, el grado de asistencia a clase, teniendo mayores posibilidades de éxito los estudiantes que asisten con mayor frecuencia.

Otros autores (Oliver et al., 2013) coinciden en que la asistencia y la participación tienen una especial trascendencia, llegando a influir en aspectos como la motivación respecto a la carrera. Resulta de especial interés la investigación de Llibrer y Latorre (2016) sobre la influencia de la asistencia a clase en el rendimiento académico. Por todas estas razones, los autores mencionados recomiendan favorecer la asistencia a clase y la participación activa en la misma; una variable que cobra especial importancia en el Espacio Europeo de Educación Superior.

A partir de estos estudios surgen, a su vez, una serie de medidas o estrategias con objeto de disminuir esta tasa de abandono universitario. Tienen especial interés las propuestas relacionadas con funciones orientadoras y tutoriales (Bernardo et al., 2015; Martin Romera et al., 2020), destacándose algunas experiencias innovadoras como los programas de coaching educativo para realizar el acompañamiento de estudiantes (Domínguez Martín et al., 2018; Sánchez Mirón y Boronat Mundina, 2014).

Muchas investigaciones apuestan por la implementación de estrategias basadas en el uso de tecnologías de información y comunicación (TIC) para mejorar el rendimiento académico y motivación del alumnado, sobre todo en los primeros cursos de carrera (Martínez-Berruezo y García-Varela, 2013). El uso de herramientas como blogs, wikis, redes sociales, marcadores, etiquetado social y microblogging entre otras, están creando nuevas situaciones de aprendizaje con un mayor índice de colaboración, personalización e inteligencia colectiva entre el alumnado (Ruiz-Palmero et al., 2013).

Cada vez es más frecuente el empleo de este tipo de herramientas en la educación superior. En los últimos años se ha extendido la integración de la docencia presencial con otros medios online. Tal es el caso de los cursos MOOC y SPOC (del inglés small private online courses) (López de la Serna et al., 2018). Una de las ventajas de estos es su potencialidad de ayudar a la formación de los colectivos en riesgo de exclusión social, más vulnerables al abandono de estudios universitarios (Vázquez Cano et al., 2018).

Sánchez-López et al. (2019) hacen una propuesta pedagógica innovadora como solución a la desmotivación del alumnado, consistente en el uso de plataformas tecnológicas y sociales del ámbito de la comunicación en el ámbito educativo, las plataformas "com-educativas". Y enfatizan la importancia del binomio creatividad-comunidad en el proceso de aprendizaje que ofrece esta herramienta.

El trabajo de investigación desarrollado por Martínez (2009) señala la necesidad de preparar la entrada a la universidad, incidiendo en la importancia del papel que juega la propia institución en la preparación de la inserción de estudiantes de bachillerato o de ciclos formativos de grado superior en sus aulas. En palabras de Martínez (2009, p. 82) “más allá de las campañas de marketing o de promoción de estudios, la colaboración y el trabajo articulado-en red- con los centros de educación secundaria es una realidad cada día más demandada y extendida". 
Asimismo, de este último estudio se desprende la importancia de que el alumnado de Educación Secundaria se familiarice con la oferta académica de una forma activa y colaborativa entre alumnado y entorno universitario, aumentando las garantías de una buena transición entre los diferentes ciclos formativos. En la actualidad, se pueden encontrar ejemplos muy puntuales de universidades españolas que incorporan el desarrollo de talleres y actividades prácticas en sus planes de orientación preuniversitaria, entre las que se destacan los programas de orientación preuniversitaria de la Universidad del País Vasco (UPV, n.d.), la universidad catalana Ramón Llull (URL, n.d.) y algunas experiencias puntuales desarrolladas en la Universidad Politécnica de Valencia (García Félix et al,, 2014), o la Universidad de Málaga ("Talleres GuíaMe-AC-UMA. Encuentros con la ciencia," 2019).

Las diferentes instituciones han desarrollado programas de acceso a la universidad que, además de ofrecer actividades más convencionales como ferias, jornadas de puertas abiertas y sesiones informativas, integran una serie de actividades prácticas organizadas, según titulaciones, para el alumnado y profesorado de centros de secundaria.

Esta investigación tiene como objetivo profundizar en la colaboración entre centros de educación secundaria y centros universitarios, incidiendo en nuevos modelos colaborativos de innovación y creatividad. La aportación más importante se traduce en mostrar una experiencia real de talleres prácticos e interactivos entre el alumnado de altas capacidades de centros escolares y estudiantes y profesores de la titulación de Arquitectura de la Escuela de Arquitectura de la Universidad de Málaga. Los objetivos de los talleres son introducir al alumnado en el campo de conocimiento de la Arquitectura, explicar la importancia de los estudios en la sociedad actual y despertar vocaciones entre otros.

Los talleres realizados han permitido a los estudiantes de institutos y colegios acercarse a dicha disciplina de una forma más dinámica e interactiva que a través de la forma que normalmente se ofrece, basada exclusivamente en la oferta de charlas informativas y jornadas de puertas abiertas.

\section{METODOLOGÍA}

La experiencia de los talleres interactivos "Nuevos retos en la Arquitectura. Taller y excursión: la ciudad como laboratorio de aprendizaje" se ha realizado por primera vez, dentro de la titulación de Arquitectura, en el curso académico 2016-2017, incluyéndose dentro del programa de mentoría universitaria GuíaMe-AC-UMA, de la Universidad de Málaga. Ha contado con la participación de doce estudiantes de bachillerato, dos de la Escuela de Arquitectura, de $4^{\circ}$ y $5^{\circ}$ curso respectivamente, dos docentes del centro, así como un mentor del mencionado programa.

El principal objetivo planteado ha sido el de acercar al alumnado de educación secundaria a los retos y salidas profesionales de los profesionales de la arquitectura en la sociedad, así como introducir conceptos básicos de la arquitectura y el urbanismo, a la forma de enseñarlos en la Escuela de Arquitectura de Málaga. En último término, se ha tratado de despertar vocaciones e interés hacia la disciplina de la Arquitectura entre el alumnado.

Con estas bases, se ha establecido una metodología de trabajo dividida en dos jornadas; la primera de ellas, en la propia escuela, y la segunda, fuera del centro, en diferentes localizaciones de la ciudad de Málaga. En un primer taller, realizado junto a los estudiantes de arquitectura, se ha pretendido acercar a los jóvenes de bachillerato a la disciplina de una forma práctica y experimental, implicándoles en un debate y resolución de un problema de forma colaborativa (figura 1). 
Se les propuso la construcción en equipo de una maqueta de ciudad ideal, con la ayuda de los estudiantes de Arquitectura, en el laboratorio de maquetas y fabricación digital del Centro. Con este ejercicio práctico se trató de potenciar su motivación y curiosidad hacia la arquitectura de una forma activa, así como estimular su creatividad (figura 2).

En una segunda sesión, y con el objetivo de ofrecer una visión amplia de la disciplina arquitectónica, se ha llevado a cabo una visita a diferentes partes de la ciudad mostrando a los estudiantes visiones de esta poco comunes en la vida diaria y cotidiana de la ciudadanía, como son las cubiertas y tejados de algunos edificios emblemáticos de la ciudad, al mismo tiempo, ejemplos de una arquitectura de referencia (figura 3).

Con ello se ha tratado de despertar en los jóvenes nuevas curiosidades, preguntas y abrir líneas de experimentación. La actividad o visitas a diferentes localizaciones se ha complementado con la realización

Figura 1. Taller práctico (fase 1) realizado en la Escuela de Arquitectura con alumnado de educación secundaria y de la titulación de Graduado en Arquitectura

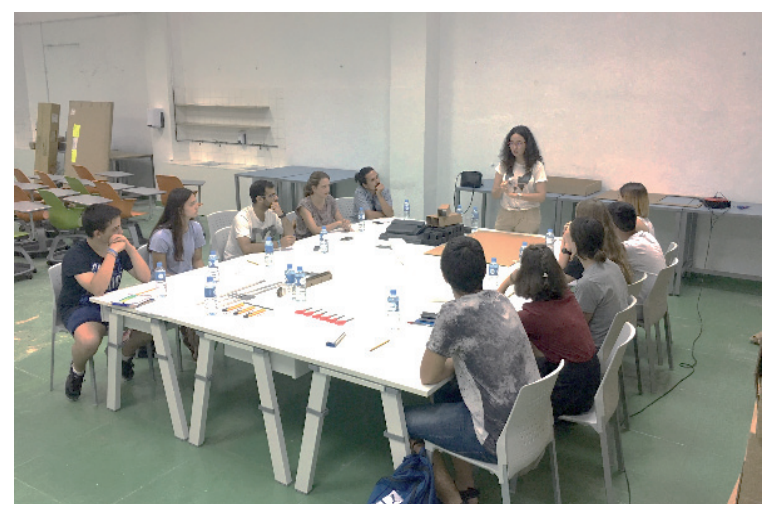

Figura 2. Realización de una maqueta de "ciudad ideal"
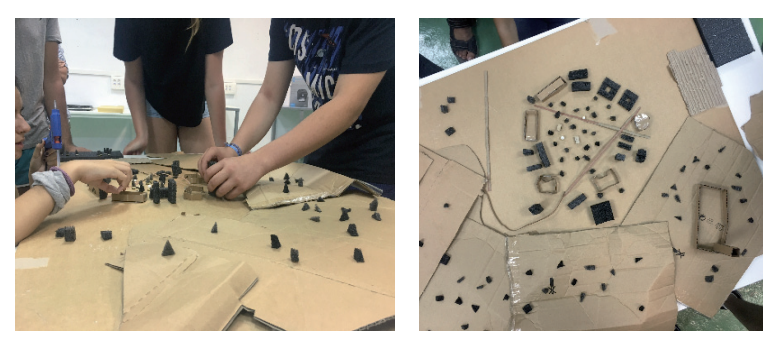

Figura 3. Visita a la cubierta de la catedral de Málaga como actividad integrada en el taller práctico (fase 2)

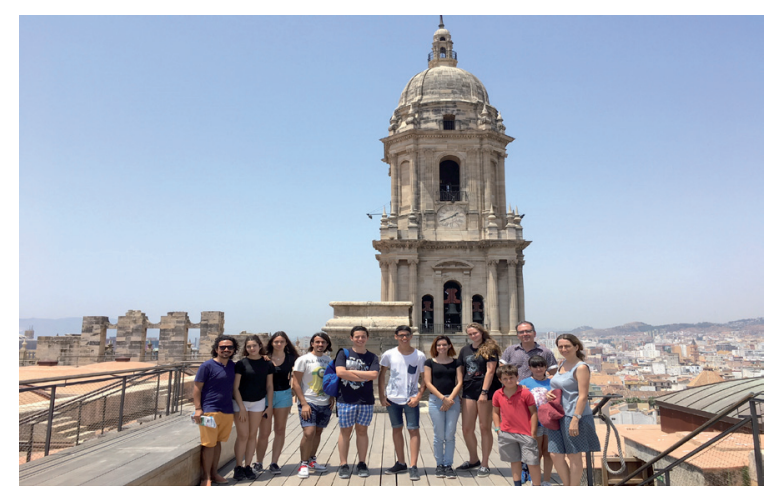

de un concurso de fotografía, en el que los participantes debían observar con atención y fotografiar visiones inusuales de la ciudad.

\section{DISCUSIÓN Y RESULTADOS}

El planteamiento de ambas sesiones se orienta a acercar al alumnado a la arquitectura de una forma experimental e interactiva, tratando de establecer un proceso en que los jóvenes sean agentes activos, a diferencia de otras fórmulas más convencionales (charlas informativas o jornadas de puertas abiertas) en que, generalmente, reciben una información como meros sujetos pasivos.

En el primer taller práctico, la interacción con el alumnado de secundaria se produce a través del debate y la pregunta abierta: "iqué lugares debería tener una ciudad ideal?, ¿qué sitios de tu barrio son los que más frecuentas y por qué?, ¿dónde juegas con los vecinos?, etcétera", tratando de fomentar un aprendizaje por descubrimiento. A partir de sus respuestas y una puesta en común de todas ellas, se lleva a cabo la construcción de una maqueta de ciudad ideal, ayudados por los estudiantes de Arquitectura. Esta se construye con los elementos que han identificado como esenciales para una ciudad amable: parques, zonas verdes, plazas y otros equipamientos y servicios como colegios, centros de deporte y salud...

Se trata de una propuesta práctica que les permite conocer y debatir sobre los aspectos fundamentales del entorno en que viven, fomentando en 
ellos una actitud crítica hacia dicho entorno y ciudad. A través de esta experiencia se persigue la obtención de un aprendizaje significativo, mediante el uso de una metodología activa, donde el profesorado se convierte en facilitador del proceso y el alumnado en agente activo que construye su propio conocimiento.

Por otro lado, se plantea un método de resolución de problemas y casos de forma colaborativa, como es el de formalizar la maqueta entre todos los participantes, que han de ponerse de acuerdo y organizarse el trabajo a realizar en un tiempo limitado. Con ello, se pretende acercar al alumnado a la forma de trabajar en la escuela de Arquitectura de Málaga, las escuelas de Arquitectura en general y a la realidad profesional del profesional de la arquitectura hoy en día, donde la colaboración con otros compañeros y equipos multidisciplinares resulta esencial.

Esta metodología de trabajo en equipo persigue fomentar el aprendizaje colaborativo, promover una actitud positiva y activa entre los componentes del grupo, aumentar el rendimiento académico y favorecer el aprendizaje significativo y autodirigido (Martínez Ramón y Gómez Barba, 2010).

Asimismo, se plantea una dinámica de trabajo que requiere una actitud creativa por parte del alumnado al tener que formalizar un nuevo modelo de ciudad. De esta forma creatividad, reflexión y aprendizaje están estrechamente unidos, se alimentan y complementan como sugiere Balderrama (2005).

En el segundo de los talleres, se persigue la interacción con los jóvenes a través de la búsqueda de nuevos puntos de vista de la ciudad, estimular en ellos nuevas curiosidades hacia la arquitectura y su entorno. La visita a la ciudad desde "lo alto" de las cubiertas de algunos edificios les ayuda a entender la complejidad de la ciudad y la diversidad de situaciones; la necesidad de acercarse a la realidad desde diferentes puntos de vista. Asimismo, el concurso de fotografía se convierte en una herramienta muy útil que les lleva a centrar su atención en aspectos concretos del entorno, aparte de identificar y filtrar la información para elaborar un resultado propio.

Los resultados recogidos en la encuesta realizada a los participantes y recomendaciones de los mentores han sido muy satisfactorios, en especial en la primera fase de la experiencia, donde las muestras del proceso creativo se hacen evidentes para los participantes de forma manifiesta. Algunos trasladan al profesorado un interés creciente hacia la arquitectura tras la realización de los talleres.

De las recomendaciones realizadas por los participantes se destaca la posibilidad de conectar en futuros talleres los contenidos trabajados con los que los jóvenes están cursando en los centros escolares. De esta recomendación se plantea la posibilidad de integrar docentes de secundaria dentro de la propia experiencia de talleres creativos.

Los mentores del programa recomendaron finalizar las sesiones con un pequeño resumen de la actividad, incidiendo en los aspectos aprendidos durante la misma. De esta sugerencia se plantea la posibilidad de incorporar una reflexión final, con la participación de los estudiantes, en que se vuelvan a plantearse los objetivos expuestos al inicio del taller. De esta forma, se adquiere una mayor conciencia de las competencias y conocimientos adquiridos, y aspectos que no han quedado claros.

Entre las sugerencias realizadas también se incluye una mayor atención a la gestión del tiempo dedicado a cada una de las actividades. Sin embargo, una de las quejas expuestas ha sido el tiempo de espera para entrar a uno de los edificios visitados, algo que no depende directamente de los docentes, sino de terceros agentes.

Los resultados satisfactorios de la experiencia, con una valoración de 9 sobre 10 por parte del alumnado, nos llevan a pensar en la posibilidad de trasladarla a un colectivo de estudiantes más amplio y no únicamente a alumnado de altas capacidades. Si entendemos esta colaboración entre centros escolares y 
universitarios como un recurso para preparar la entrada en las aulas de la universidad, resulta vital integrar, dentro del programa, a un mayor número de estudiantes.

Algunas universidades ya han tomado conciencia de esta necesidad, destacándose la labor realizada por la Universidad del País Vasco, que abre sus actividades prácticas de orientación a todo el alumnado de primer curso de Bachillerato, Ciclos Formativos de Grado Superior de Formación Profesional, Enseñanzas Artísticas y Enseñanzas Deportivas de la Comunidad Autónoma Vasca, y al profesorado y personal orientador de centros de secundaria.

Cuenta, además, con una amplia oferta de actividades relacionadas con un alto número de titulaciones en el curso académico 2017-2018 (Administración y Dirección de Empresas, Antropología Social, Química, Ingeniería Informática, Ingeniería Eléctrica, Ingeniería Civil, Ingeniería en Energías Renovables, Ingeniería Electrónica Industrial y Automática, Ingeniería Mecánica, Arquitectura Técnica y Fundamentos de Arquitectura).

Curiosamente, según los datos extraídos del Informe Datos y Cifras del Sistema Universitario Español, del curso 2015-2016, la tasa de abandono de grado por comunidades autónomas sitúa al País vasco, junto a Navarra y Castilla y León, entre las tres con una menor tasa, del 17,4\%, 16,9 \% y 16,0 \% respectivamente (Ministerio de Educación, 2016).

En el caso de la experiencia realizada en la escuela de Arquitectura de Málaga resulta difícil cuantificar su impacto en la tasa de abandono de los estudios de Arquitectura. Para ello, sería necesario incrementar el número de participantes y repetirla durante dos o tres años, de forma que permita comparar dicha tasa de abandono en diferentes cursos académicos.

\section{CONCLUSIONES}

A partir de las investigaciones previas realizadas en torno al abandono de los estudios universitarios por parte del alumnado, se deduce el papel fundamental que juega la universidad en la preparación de la inserción de estudiantes de bachillerato o de ciclos formativos de grado superior en sus aulas. Sin embargo, son instituciones muy puntuales las que han tomado conciencia de esta realidad en España y han incorporado medidas preventivas entre sus prácticas. Algunas de ellas, como la Universidad de Málaga, ha iniciado una serie de talleres prácticos que permiten investigar en esta línea de colaboración entre centros escolares y universitarios.

La experiencia de talleres creativos realizados en Arquitectura ha permitido dar respuesta a los objetivos inicialmente planteados: introducir al estudiante en el campo de conocimiento de la Arquitectura, explicar la importancia de los estudios en la sociedad actual y despertar vocaciones. Para ello se ha planteado una metodología de talleres basada en las siguientes características:

\subsubsection{Talleres interactivos:}

Las dos fases planteadas han sido planificadas tratando de establecer un proceso en que los jóvenes sean agentes activos, a diferencia de otras fórmulas más convencionales (charlas informativas o jornadas de puertas abiertas) en que se limitan a recibir la información como meros sujetos pasivos. Se ha tratado de fomentar una metodología activa, donde los docentes y mentores se han convertido en facilitadores del proceso, y el alumnado, el agente activo que ha construido su propio conocimiento. 
- Talleres colaborativos. El planteamiento de la dinámica de trabajo en equipo, especialmente en la primera parte de talleres, persigue fomentar el aprendizaje colaborativo, promover una actitud positiva y activa entre los miembros del grupo y, en último término, potenciar un aprendizaje significativo.

- Talleres creativos. Las prácticas planteadas por el profesorado, tanto la elaboración de un nuevo modelo de ciudad como el concurso de fotografía de visiones urbanas no cotidianas, requieren una actitud creativa por parte de los participantes. Los procesos llevados a cabo integran creatividad, reflexión (ambas actividades exigen un análisis y valoración de elementos y contexto) y aprendizaje.

Por otro lado, y por tratarse de una primera experiencia, el diseño de la metodología puede mejorarse en diferentes aspectos. Siguiendo algunas de las recomendaciones de encuestas y valoraciones de mentores, las posibles mejoras estarían orientadas a:

- La incorporación de docentes y orientadores de educación secundaria y ciclos formativos.

- La optimización en la gestión del tiempo destinado a cada actividad planteada.

- La incorporación de resúmenes y reflexiones para finalizar cada actividad, haciendo hincapié en capacidades y competencias trabajadas.

Por último, cabría señalar que la experiencia de talleres realizada no permite, por el momento, valorar cuantitativa ni cualitativamente el impacto ni en tasas de abandono de la titulación de Arquitectura ni en el interés que ha despertado en el alumnado. Para ello, sería necesario incrementar el número de participantes en talleres y repetirlos durante dos o tres años, de forma que permita comparar resultados entre el alumnado en diferentes cursos académicos de la escuela de Arquitectura de Málaga. No obstante, esta experiencia ha permitido abrir una nueva línea de investigación para seguir profundizando en la colaboración entre los diferentes ciclos educativos.

\section{REFERENCIAS}

Araque, F., Roldán, C., y Salguero, A. (2009). Factors influencing university drop out rates. Computers and Education, 53(3), 563-574.

Balderrama, M. (2005). Creatividad y Aprendizaje. Lima.

Bernardo, A. B., Cerezo, R., Núñez, J. C., Tuero, E., y Esteban, M (2015). Predicción del abandono universitario: variables explicativas y medidas de prevención. Revista Fuentes, 16, 63-84.

Corominas Rovira, E. (2001). La transición a los Estudios Universitarios. Aandono o cambio en el primer año de Universidad. Revista de Investigación Educativa, 19(1), 127-151.

Datos básicos del sistema universitario español. Curso 2013-2014. Catálogo de publicaciones del Ministerio: mecd.gob.es. (2013). https://www.mecd.gob.es/prensa-mecd/dms/mecd/prensamecd/actualidad/2014/02/20140213-datos-univer/datoscifras-13-14.pdf
Domínguez Martín, R., Cruz Chust, A. M., y Ferrando Rodríguez, M. L. (2018). Implementando el coaching educativo en la universidad virtual, una herramienta de desarrollo personal. Innoeduca. International Journal of Technology and Educational Innovation, 4(2) https://doi.org/10.24310/Innoeduca.2018.V4i2.4930.

García Félix, E., Conejero Casares, J. A., y Díez Ruano, J. L. (2014). La entrada en la universidad: un reto para la orientación académica. Revista de Docencia Universitaria-REDU, 12(2), 255-280.

Llibrer Escrig, I., y Latorre Guillem, M. Á. (2016). Asistencia a clase en el espacio europeo de educació superior. Opción, 12, 1052-1074.

López de la Serna, A., Castaño Garrido, C., y Herrero Fernández, D. (2018). Integración de los cursos SPOC en las asignaturas de 
grado. Una experiencia práctica. Píxel-Bit. Revista de Medios y Educación, 52, 139-149.

Martín Romera, A., Berrios Aguayo, B., y Pantoja Vallejo, A. (2020). Factores y elementos de calidad percibidos por el profesorado participante en el plan de acción tutorial de universidades europeas. Educación XX1, 23(1), 349-371. https://doi. org/10.5944/educxx1.23874

Martínez, M. (2009). La orientación y la tutoría en la universidad en el marco del Espacio Europeo de Educación Superior (EEES). Revista Fuentes, 9, 78-97.

Martínez-Berruezo, M. A., y García-Varela, A. B. (2013). Volver Análisis de la influencia de la virtualización en la motivación de alumnado universitario de primer curso de Magisterio. Revista de Educación, 362, 42-68. https://doi.org/10.4438/1988592X-RE-2011-362-152

Martínez Ramón, J. P., y Gómez Barba, F. (2010). La técnica puzzle de Aronson: descripción y desarrollo. In P. . Arnaiz \& F. J. (Coords. . Hurtado, Ma.D. y Soto (Eds.), 25 Años de Integración Escolar en España: Tecnología e Inclusión en el ámbito educativo, laboral y comunitario. Consejería de Educación de Murcia.

Ministerio de Educación, C. y D. (2016). Datos y Cifras del sistema universitario español. Curso 2015 2016. Catálogo de Publicaciones Del Ministerior: Mecd.gob.es. https://www.mecd.gob. es/dms/mecd/servicios-al-ciudadano-mecd/estadisticas/ educacion/universitaria/datos-cifras/datos-y-cifras-SUE2015-16-web-.pdf

Oliver, A, Vivo, J., y Galiana, L. (2013). Determining Asessment Performance in Applied Statistics with ROC Analysis. The UB Journal of Psychology, 43(3), 363-379.

Ruiz-Palmero, J., Sánchez-Rodríguez, J., y Gómez García, M. (2013). Entornos personales de aprendizaje: estado de la situación en la Facultad de Ciencias de la Educación de la Universidad de Málaga. Pixel-Bit. Revista de Medios y Educación, 42, 171-181.

Sánchez-López, I., Pérez-Rodríguez, A., y Fandos-Igado, M. (2019). Com-educational Platforms: Creativity and Community for Learning. Journal of New Approaches in Educational Research, 8(2), 214-226. https://doi.org/10.7821/naer.2019.7.437
Sánchez Mirón, B., y Boronat Mundina, J. (2014). Coaching educativo: modelo para el desarrollo de competencias intra e interpersonales. Educación XX1, 17(1), 221-242. https://doi. org/10.5944/educxx1.17.1.10712

Talleres GuíaMe-AC-UMA. Encuentros con la ciencia. (2019). https://www.encuentrosconlaciencia.es/?cat=38

UPV. (n.d.). Programas de Acceso a la universidad de la Universidad del País Vasco. Retrieved December 8, 2019, from http:// www.url.edu/es/estudios/nuevos-estudiantes/talleresorientacion-profesional

URL. (n.d.). Talleres de Orientación Profesional en la Universidad Ramón Llull. Retrieved December 8, 2019, from http://www. url.edu/es/estudios/nuevos-estudiantes/talleres-orientacion-profesional

Vázquez Cano, E., López Meneses, E. J., y Martín Padilla, A. H. (2018). Los Nuevos Entornos Virtuales De Aprendizaje Permanente (MOOC). Un Estudio diacrónico Del Estudiantado De La Universidad Pablo De Olavide (2015-2017). EDMETIC, 7(1), 350-371. https://doi.org/https://doi.org/10.21071/edmetic. v7i1.10080

Willcoxson, L. (2010). Factors affecting intention to leave in the first, second and third year of university studies: a semesterby-semester investigation. Higher Education Research and Development, 29(6), 623-639.

\section{Agradecimientos}

Este artículo se ha construido a partir de los resultados obtenidos en los talleres realizados dentro del programa de Investigación y mentoría universitaria GuíaMe-AC-UMA para alumnado de altas capacidades de bachillerato y ciclos formativos, cuyo investigador principal es Enrique Viguera Minguez. 